\title{
Relationship of Maximum Rate of Pressure Rise Between Aorta and Left Ventricle in Pediatric Patients Implication for Ventricular-Vascular Interaction With the Potential for Noninvasive Determination of Left Ventricular Contractility
}

\author{
Satoshi Masutani, MD; Yoichi Iwamoto, MD; \\ Hirotaka Ishido, MD; Hideaki Senzaki, MD
}

\begin{abstract}
Background: The maximum rate of the ventricular pressure rise (dp/dtmax) provides a reliable measure of ventricular contractility. However, its estimation requires invasive measurement of left ventricular (LV) pressure, limiting its bedside clinical applicability. In the present study, 2 hypotheses were tested: (1) that the ratio of $\mathrm{dp} / \mathrm{dtmax}$ between the aorta (Ao) and LV is consistent among patients despite marked differences in underlying cardiac disease, contractile state and heart rate when vascular mechanical and loading properties are taken into account, and (2) that using such a relationship, LV dp/dtmax can be estimated from Ao dp/dtmax, potentially providing a method of noninvasive determination of LV contractility.

Methods and Results: Data from 30 control children and 45 pediatric patients with various cardiovascular diseases revealed that the characteristic impedance $(\mathrm{Zc})$ and mean arterial pressure were significant determinants of the Ao-LV dp/dtmax relationship in both control and disease groups. LV dp/dtmax estimated using the regression

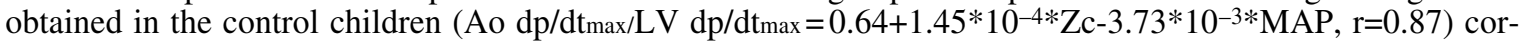

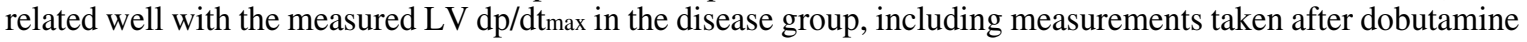
and atrial pacing $(\mathrm{r}=0.89)$.

Conclusions: Ao dp/dtmax and LV dp/dtmax are closely correlated through the vascular loading properties and $\mathrm{LV}$ dp/dtmax can be derived from Ao dp/dtmax, which has potential as a noninvasive method of determining LV contractility. (Circ J 2009; 73: 1698-1704)
\end{abstract}

Key Words: Contractility; Diagnosis; Left ventricular function

A sessing the ventricular contractile state, coupled with vascular loading conditions, is important for better understanding of the pathophysiology of various cardiovascular diseases. The maximum rate of the ventricular pressure rise $(\mathrm{dp} / \mathrm{dt} \max )$ provides a simple but reliable measure of ventricular contractility. ${ }^{1}$ Despite its apparent preload dependence, ${ }^{2}$ this index generally reflects the ventricular contractile state irrespective of ventricular morphology, localized wall motion abnormalities or structural abnormalities, ${ }^{3}$ and parallels the change in contractility assessed by load independent indexes in both acute and chronic conditions. ${ }^{4,5}$ Thus, ventricular $\mathrm{dp} / \mathrm{dtmax}$ has been extensively used for the assessment of ventricular contractile function in a variety of experimental ${ }^{6,7}$ and clinical ${ }^{8}$ settings. However, determination of this index requires invasive measurement of ventricular pressure by intraventricular catheterization, thus limiting its bed-side clinical applicability.

Blood flow ejected from the left ventricle (LV) into the arterial system generates aortic pressure depending on the interaction betweenthe ventricular and vascular properties. Increased blood flow induced by increased LV contractility

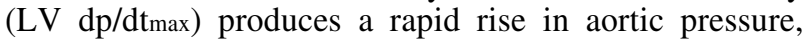
whereas changes in arterial properties under constant blood flow alter the way in which the aortic pressure develops. Therefore, it is highly possible that the maximum rate of aortic pressure rise (Ao dp/dtmax) and LV dp/dtmax could have an intrinsic correlation that depends on the vascular mechanical and loading properties. If the relationship between Ao dp/dtmax and LV dp/dtmax is quantitatively determined, then the LV dp/dtmax can be calculated from Ao $\mathrm{dp} / \mathrm{dtmax}$, potentially providing a method of noninvasively estimating LV contractility.

The present study was conducted to test 2 hypotheses: (1) that the ratio between Ao dp/dtmax and LV dp/dtmax is consistent among patients despite marked differences in underlying cardiac disease, contractile state and heart rate (HR) when vascular mechanical and loading properties are taken into account, and (2) that based on such a relationship, $\mathrm{LV}$ dp/dtmax can be accurately estimated from Ao dp/dtmax.

(Received October 9, 2008; revised manuscript received April 6, 2009; accepted April 13, 2009; released online July 13, 2009)

Department of Pediatric Cardiology, International Medical Center, Saitama Medical University, Saitama, Japan

Mailing address: Hideaki Senzaki, MD, Department of Pediatric Cardiology, International Medical Center, Saitama Medical University,

Staff Office Building 303, 1397-1 Yamane, Hidaka, Saitama 350-1298, Japan. E-mail: hsenzaki@saitama-med.ac.jp

All rights are reserved to the Japanese Circulation Society. For permissions, please e-mail: cj@j-circ.or.jp 


\section{Methods}

\section{Patients}

The study involved 75 pediatric patients who underwent diagnostic/interventional cardiac catheterization at Saitama Medical University Hospital. Of them, 30 patients who had recovered after Kawasaki disease and had normal coronary angiography served as a control group to quantify the determinants of the Ao dp/dtmax and LV dp/dtmax relationship. The remaining patients $(n=45$, disease group) had various cardiovascular diseases with ventricular anatomical and/or functional abnormalities (Table 1). None of the study patients had aortic valvular disease or LV outflow obstruction, which could potentially affect the LV-Ao pressure interaction. Kawasaki disease patients showed normal findings on routine check-up before catheterization (ECG, chest X-ray, laboratory examination, echocardiographic assessment for cardiac function and structure). Written informed consent was given by all parents, and the study was approved by the Saitama Medical University Ethical Committee.

\section{Procedures}

All measurements were performed during routine cardiac catheterization before angio/ventriculography. The LV and Ao pressures were measured using a high-fidelity micromanometer mounted on a 0.014 -inch guidewire ${ }^{9}$ with the guidewire drawn back from the LV to ascending Ao. Ascending aortic flow was measured simultaneously using a flow velocity probe..$^{10}$

To further test the influence of changes in contractile state and HR on the Ao dp/dtmax and LV dp/dtmax relationship, measurements were repeated after increased contractility by dobutamine $\left(5 \mu \mathrm{g} \cdot \mathrm{kg}^{-1} \cdot \mathrm{min}^{-1}\right.$, in a randomly selected 22 and 32 patients from the control and disease groups, respectively) or after increased HR by atrial pacing (160 180 beats $/ \mathrm{min}$, randomly selected 14 patients in the disease group). All data were digitized at $500 \mathrm{~Hz}$ and stored for subsequent off-line analysis.

\section{Data Analysis}

The LV dp/dtmax and Ao dp/dtmax were calculated using the 5-point weighted method and averaged for 2-4 consecutive beats during the expiratory phase. These calculations were performed by automated algorithm to ensure accuracy and reproducibility. Vascular properties were calculated using Fourier components of pressure and flow data as reported previously. ${ }^{11}$ Briefly, total arterial resistance (Rt) was calculated by subtracting the mean right atrial pressure from the mean ascending aortic pressure (MAP), and dividing the difference by cardiac output. Characteristic impedance (Zc), a measure of proximal aortic stiffness, was calculated by averaging all impedance moduli between 2 and $10 \mathrm{~Hz}$. Total arterial compliance (C) was calculated using the method reported by Liu et al. ${ }^{12}$

\section{Statistical Analysis}

The baseline characteristics of the control and disease groups were compared using 2-tailed unpaired t-test. We assessed the determinants of the Ao dp/dtmax-LV dp/dtmax ratio by using multiple stepwise linear regression analysis with $\mathrm{Zc}$, Rt, C, HR, and MAP included as independent variables. To explore the validity of regression analysis for the data of a clinically relevant broader range of contractility, both data at rest and those for dobutamine-induced increased contractility were combined for analysis. In the disease group, data
Table 1. Diagnoses in the Disease Group

\begin{tabular}{lr}
\hline Diagnosis & $\mathrm{n}$ \\
\hline Atrial septal defect & 13 \\
Ventricular septal defect & 17 \\
Isolated right ventricular hypoplasia & 1 \\
Primary pulmonary hypertension & 1 \\
Peripheral pulmonary stenosis & 2 \\
Hypertrophic cardiomyopathy & 1 \\
Postoperative state of total anomalous pulmonary & 1 \\
$\quad$ venous connection & \\
Postoperative state of tetralogy of Fallot (all had residual & pulmonary stenosis and pulmonary regurgitation) \\
Postoperative state of ventricular septal defect (1 had & 6 \\
pulmonary hypertension; 2 had pulmonary valvular stenosis) & 3 \\
\hline
\end{tabular}

Table 2. Baseline Characteristics of Control and Disease Group Subjects

\begin{tabular}{|c|c|c|}
\hline & $\begin{array}{l}\text { Control group } \\
(\mathrm{n}=30)\end{array}$ & $\begin{array}{l}\text { Disease group } \\
\quad(\mathrm{n}=45)\end{array}$ \\
\hline \multicolumn{3}{|l|}{ Demographic data } \\
\hline $\mathrm{M} / \mathrm{F}$ & $21 / 9$ & $25 / 20$ \\
\hline Age, years & $\begin{array}{c}5.5 \pm 0.78 \\
(0.6-17.4)\end{array}$ & $\begin{array}{c}4.8 \pm 0.54 \\
(0.33-16)\end{array}$ \\
\hline BSA, $m^{2}$ & $\begin{array}{c}0.79 \pm 0.06 \\
(0.35-1.62)\end{array}$ & $\begin{array}{c}0.68 \pm 0.04 \\
(0.24-1.5)\end{array}$ \\
\hline \multicolumn{3}{|l|}{ Hemodynamic parameters } \\
\hline $\mathrm{CI}, \mathrm{L} \cdot \mathrm{min}^{-1} \cdot \mathrm{m}^{-2}$ & $\begin{array}{l}4.3 \pm 0.18 \\
(2.2-5.9)\end{array}$ & $\begin{array}{c}3.7 \pm 0.16 \\
(1.3-6.8)\end{array}$ \\
\hline $\mathrm{HR}$, beats/min & $\begin{array}{l}112 \pm 2.6 \\
(87-140)\end{array}$ & $\begin{array}{l}110 \pm 3.2 \\
(69-168)\end{array}$ \\
\hline SBP, mmHg & $\begin{array}{l}92.4 \pm 2.4 \\
(70-116)\end{array}$ & $\begin{array}{l}92.5 \pm 2.0 \\
(66-116)\end{array}$ \\
\hline DBP, mmHg & $\begin{array}{r}63.3 \pm 2.5 \\
(41-86)\end{array}$ & $\begin{array}{r}60.0 \pm 1.9 \\
(35-88)\end{array}$ \\
\hline MAP, mmHg & $\begin{array}{c}77.9 \pm 2.4 \\
(56-100)\end{array}$ & $\begin{array}{l}76.0 \pm 1.9 \\
(52-103)\end{array}$ \\
\hline \multicolumn{3}{|l|}{ Vascular properties } \\
\hline Rt, dynes $\cdot \mathrm{s}^{-1} \cdot \mathrm{cm}^{-5}$ & $\begin{array}{l}25.3 \pm 1.3 \\
(14.5-43.7)\end{array}$ & $\begin{array}{l}32.7 \pm 2.6^{*} \\
(11.8-94)\end{array}$ \\
\hline $\mathrm{C}, \mathrm{ml} / \mathrm{mmHg}$ & $\begin{array}{c}1.13 \pm 0.17 \\
(0.27-3.96)\end{array}$ & $\begin{array}{c}0.74 \pm 0.10 * \\
(0.13-4.10)\end{array}$ \\
\hline Zc, dynes $\cdot \mathrm{s}^{-1} \cdot \mathrm{cm}^{-5}$ & $\begin{array}{l}185 \pm 15 \\
(72-388)\end{array}$ & $\begin{array}{l}288 \pm 28 * \\
(76-1,072)\end{array}$ \\
\hline \multicolumn{3}{|l|}{ Pressure rise } \\
\hline 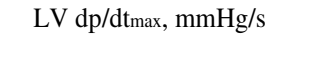 & $\begin{array}{c}1,403 \pm 68 \\
(1,051-2,574)\end{array}$ & $\begin{array}{l}1,230 \pm 60 \\
(782-2,538)\end{array}$ \\
\hline Ao dp/dtmax, $\mathrm{mmHg} / \mathrm{s}$ & $\begin{array}{l}500 \pm 11 \\
(384-610)\end{array}$ & $\begin{array}{l}534 \pm 17 \\
(293-887)\end{array}$ \\
\hline Ao dp/dtmax $/ \mathrm{LV}$ dp/dtmax & $\begin{array}{c}0.37 \pm 0.013 \\
(0.24-0.52)\end{array}$ & $\begin{array}{l}0.43 \pm 0.013 * \\
(0.24-0.59)\end{array}$ \\
\hline
\end{tabular}

Values are expressed as mean \pm SEM, and values in parentheses indicate data range. ${ }^{*} \mathrm{P}<0.05$ vs control group.

BSA, body surface area; CI, cardiac index; HR, heart rate; SBP, systolic blood pressure; DBP, diastolic blood pressure; MAP, mean arterial blood pressure; Rt, total systemic vascular resistance; $\mathrm{C}$, mean arterial compliance; $\mathrm{Zc}$, characteristic impedance; LV, left ventricular; Ao, aortic.

during atrial pacing, as well as at rest and after dobutamine infusion, were combined for analysis to further explore the possible applicability of the Ao-LV dp/dtmax relationship for a range of diseases and conditions. Correlations between the measured and estimated LV dp/dtmax were tested by Pearson's correlation coefficient. $\mathrm{P}<0.05$ was considered statistically significant. All statistical analyses were performed using JMP, version 7 (SAS Institute Inc, Cary, NC, USA). 
Table 3. Results of Stepwise Regression to Estimate the Ratio of Ao dp/dtmax to LV dp/dtmax

\begin{tabular}{lccccc}
\hline & \multicolumn{5}{c}{ P value } \\
\cline { 2 - 6 } & HR & MAP & Rt & Zc & C \\
\hline Control group & 0.4098 & $<0.0001(-0.7828)$ & 0.6719 & $0.0292(0.2270)$ & 0.5017 \\
Disease group & & & & & 0.0005 \\
$\quad$ Rest & 0.5775 & 0.0000 & 0.6797 & 0.0041 & 0.8408 \\
Dobutamine infusion & 0.8219 & 0.0001 & 0.8050 & 0.0177 & 0.9847 \\
Atrial pacing & 0.5068 & 0.0037 & 0.8050 \\
\hline
\end{tabular}

$\mathrm{P}$ values for each parameter are listed. Data in parentheses indicate standardized correlation coefficients ( $\beta$ values). Abbreviations see in Table 2 .

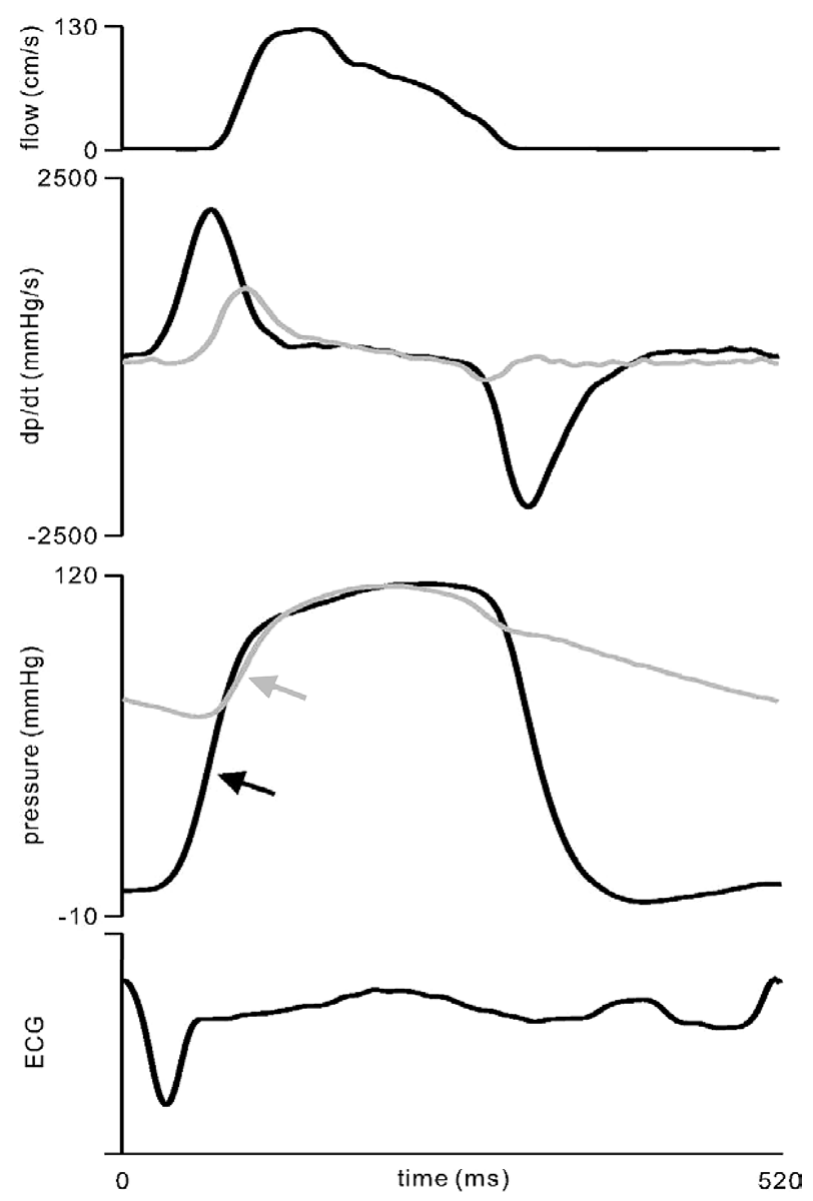

Figure 1. Time interval between left ventricular (LV) and aortic (Ao) dp/dtmax. Analog recordings of LV (black) and Ao (gray) pressures and their time derivatives (dp/dt), ECG and aortic flow are simultaneously presented. At end-diastole, LV pressure rises and LV $\mathrm{dp} / \mathrm{dtmax}$ occurs (black arrow) before the aortic valve opens during the isovolumic contraction phase. After the aortic valve opens, the aortic pressure rises as aortic flow occurs. Ao dp/dtmax occurs (gray arrow) during the early ejection period when LV dp/dt is decreasing. Thus, $\mathrm{LV}$ and Ao dp/dtmax occur in different phases before and after aortic valve opening, respectively.

\section{Results}

Table 2 summarizes the demographic, hemodynamic and impedance data of each study group. The disease group had a broad range of baseline contractility, HR and blood pressures. The pulmonary to systemic blood flow ratio (Qp/Qs) ranged from 0.79 to 4.59 (median 1.36) and the right ventricular systolic pressure ranged from 18 to 85 (median 33) $\mathrm{mmHg}$ in the disease group. There were no significant dif-

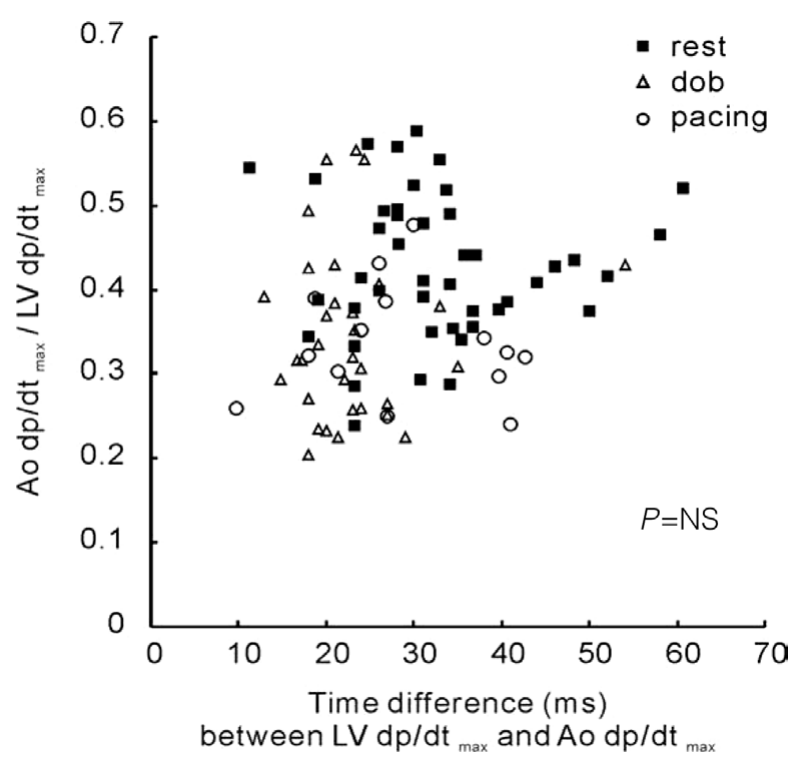

Figure 2. Impact of the time interval between left ventricular (LV) and aortic (Ao) dp/dtmax on the ratio of Ao-LV dp/dtmax. There was no significant effect: $(\boldsymbol{\square})$ at rest; $(\triangle)$ after dobutamine; $(\bigcirc)$ during atrial pacing).

ferences between the control and disease groups with regard to age, sex, body surface area, HR, and blood pressure (systolic, diastolic or mean). In contrast, the vascular properties of the disease group differed from those of the control group: higher Rt, lower $\mathrm{C}$, and higher $\mathrm{Zc}$, indicating less compliant and stiffer arterial wall properties in both the proximal and peripheral vessels. Although not statistically significant, the LV dp/dtmax was smaller and the Ao dp/dtmax was higher in the disease group than in the control group, with a resultant significantly higher Ao dp/dtmax/LV dp/dtmax ratio, compared with the control group.

\section{Determinants of the Ao dp/dtmax and LV dp/dtmax Relationship}

Table 3 summarizes the results of stepwise regression analysis. In the control group, Zc and MAP, but not $\mathrm{C}$, Rt, and HR, correlated significantly with the Ao dp/dtmax/LV $\mathrm{dp} / \mathrm{dtmax}$ ratio. Similar findings were noted in the disease group, even after taking into consideration the dobutamine infusion- and pacing state-related data. These results indicated that the Ao-LV dp/dtmax relationship can be determined by Zc and MAP, independent of the underlying disease, contractile state, and tachycardia state. The regression equation derived from the control group, including data for dobutamine infusion, is: 

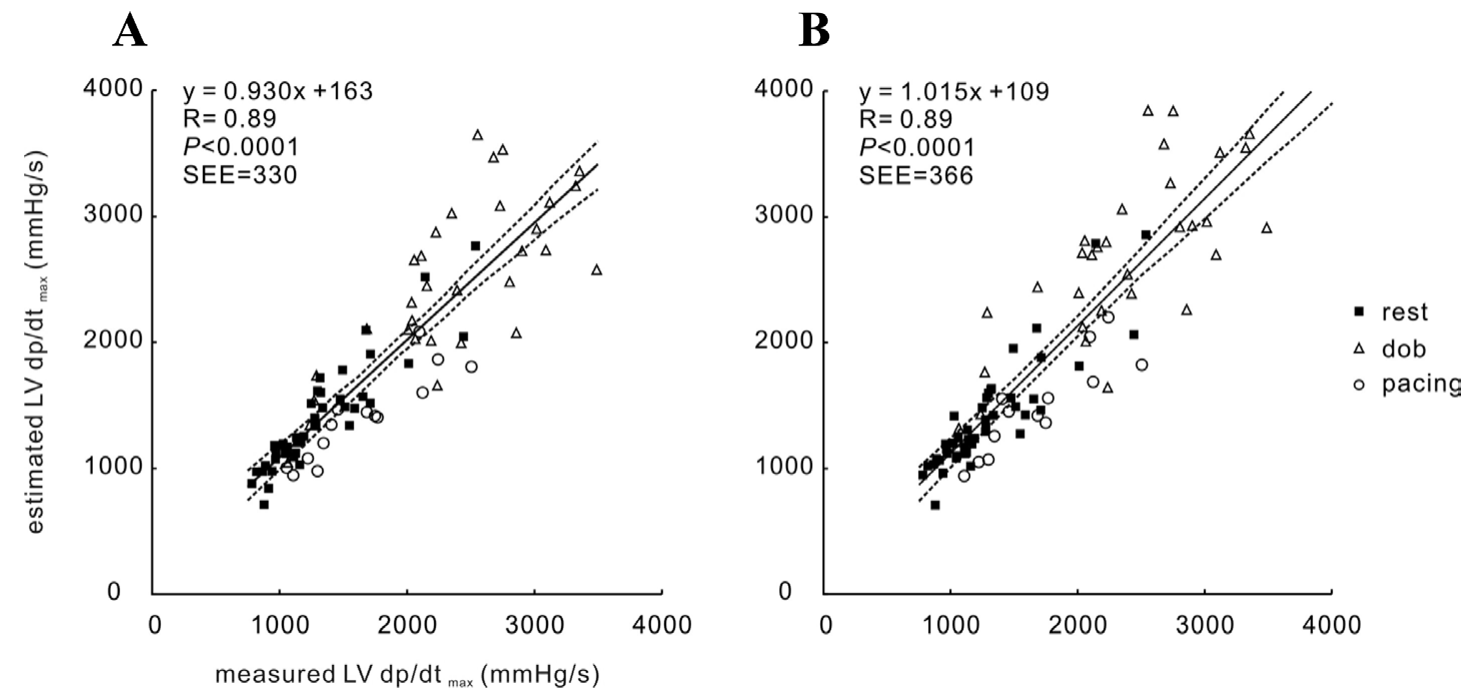

Figure 3. Correlation between measured and estimated LV dp/dtmax. Mean aortic pressure and characteristic impedance $(\mathbf{A})$ or mean aortic pressure alone $(\mathbf{B})$ were used to estimate LV dp/dtmax. $(\triangle)$ Dobutamine infusion, $(\bigcirc)$ atrial pacing. Dashed lines represent $95 \%$ confidence intervals.
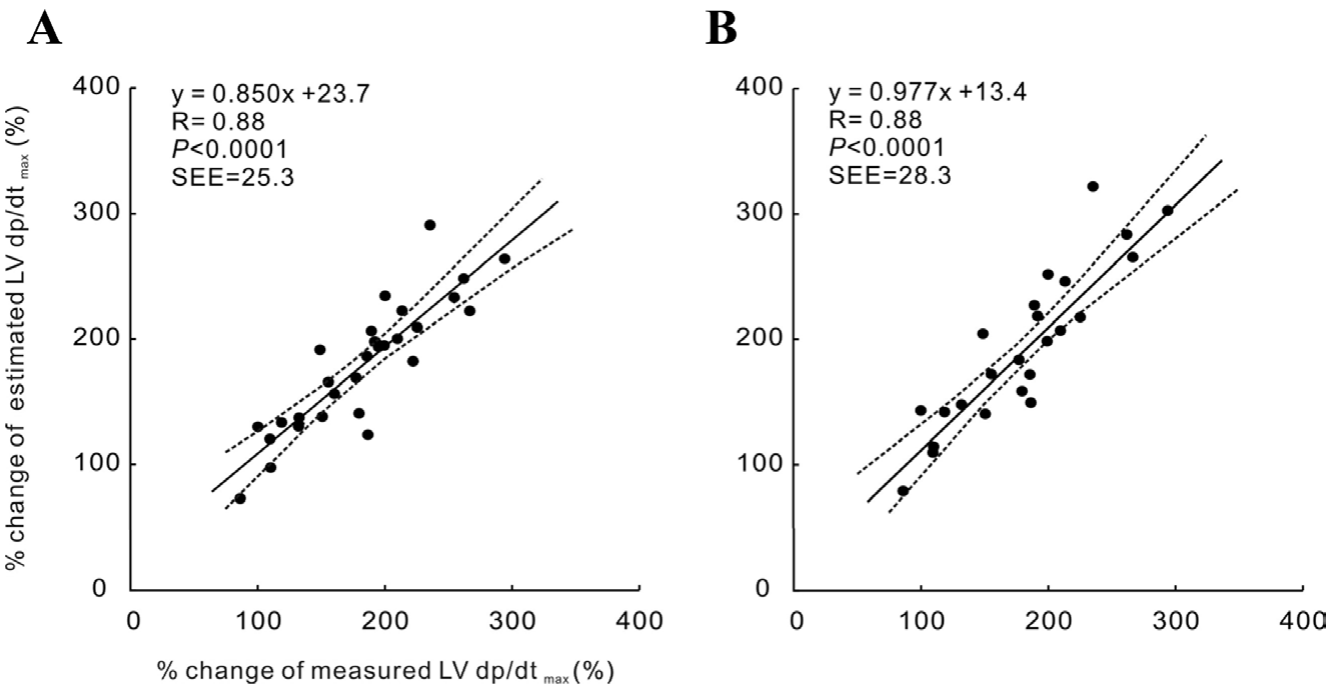

Figure 4. Correlation between measured and estimated percent changes in left ventricular (LV) dp/dtmax before and after dobutamine infusion. Mean aortic pressure and characteristic impedance $(\mathbf{A})$ or mean aortic pressure alone $(\mathbf{B})$ were used to estimate LV dp/dtmax. Dashed lines represent $95 \%$ confidence intervals.

Ao dp/dtmax $/ \mathrm{LV} \mathrm{dp} / \mathrm{dtmax}=$

$0.641+1.445^{*} 10^{-4 *} \mathrm{Zc}-3.73 * 10^{-3 *} \mathrm{MAP}$

$(\mathrm{P}<0.0001, \mathrm{R}=0.87)$.

It is possible that the time difference between Ao dp/dtmax and LV dp/dtmax (Figure 1) also affects their ratio. Therefore, we further analyzed the effects of the $\mathrm{dp} / \mathrm{dtmax}$ timing on the Ao-LV dp/dtmax relationship. The results, however, showed no significant effects (Figure 2). In patients with a ventricular septal defect (VSD), the interventricular communication during the pre-ejection phase may affect the results; however, the results did not change after the VSD patients were excluded (Figure 2).

\section{Estimation of LV dp/dtmax From Ao dp/dtmax}

The consistent Ao-LV dp/dtmax relationship among patients with different cardiovascular diseases, contractile states, and HR suggested that LV dp/dtmax could reasonably be estimated from Ao dp/dtmax. Figure 3 shows the results for 45 patients with various cardiovascular diseases, including results obtained under increased contractility with dobutamine and under increased HR by atrial pacing. There was a high correlation between the measured LV dp/dtmax and the estimated LV dp/dtmax $[y=0.930 * x+163$ ( $r=0.89$, SEE $=$ $330, \mathrm{P}<0.0001)]$. The regression lines were not significantly different among baseline, dobutamine infusion and atrial pacing ( $\mathrm{P}=\mathrm{NS}$ by ANCOVA). Because computing $\mathrm{Zc}$, but not MAP, requires a somewhat complex calculation, and because of the high statistical power of MAP in determining the Ao-LV dp/dtmax relationship, we further tested whether $\mathrm{LV} \mathrm{dp/dtmax} \mathrm{can} \mathrm{be} \mathrm{reasonably} \mathrm{estimated} \mathrm{from} \mathrm{the}$ regression equation using MAP alone without $\mathrm{Zc}$ :

$$
\text { Ao dp/dtmax }-\mathrm{LV} \text { dp/dtmax }=0.694-4.00 * 10^{-3 *} \mathrm{MAP}
$$


A

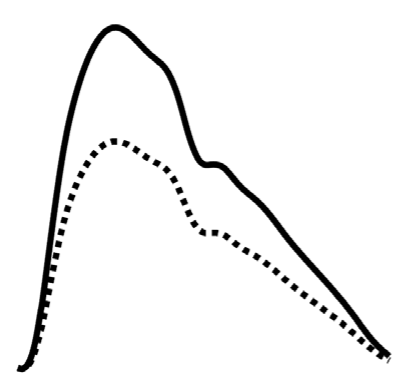

C

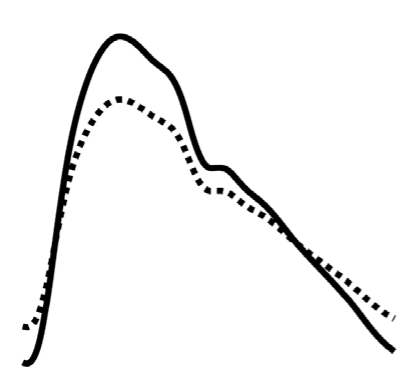

B

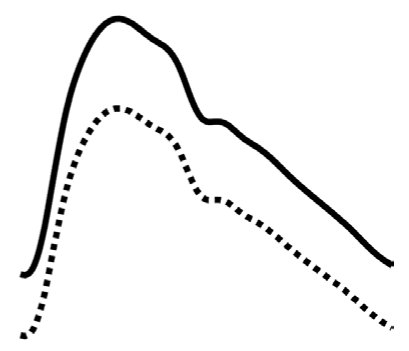

D

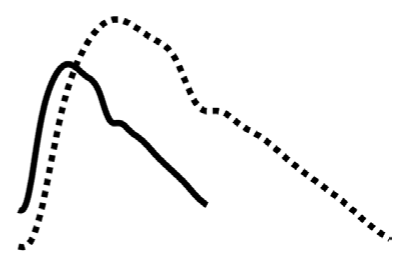

Figure 5. Qualitative simulation demonstrating the independent effects of changes in vascular properties on aortic pressure trajectory when aortic flow is kept constant. Solid lines represent aortic pressure trajectories when characteristic impedance (A), total arterial resistance $(\mathbf{B})$, total arterial compliance $(\mathbf{C})$, or heart rate $(\mathbf{D})$ are increased from the baseline state (dashed lines) under constant cardiac output. The 3-compartment (characteristic impedance, total arterial resistance and total arterial compliance) Windkessel model was applied under constant blood flow, and each property was changed by $50 \%$ of baseline values.
The results again showed a good correlation between the measured and estimated LV dp/dtmax (Figure 3B, y= $1.015 * \mathrm{x}+109, \mathrm{r}=0.89, \mathrm{SEE}=366, \mathrm{P}<0.0001)$.

Because the data collected after dobutamine infusion in each method showed a relatively higher variance than those at rest, to further test the clinical validity of our method we compared the percent changes in $\mathrm{LV} \mathrm{dp} / \mathrm{dtmax}$ before and after dobutamine infusion between the measured and estimated variables. As shown in Figure 4, the estimated LV dp/dtmax by each equation (Figure 4A by equation 1; Figure $4 \mathrm{~B}$ by equation 2) provided a similar dobutamineinduced percent change as those calculated by the measured LV dp/dtmax $(\mathrm{y}=0.850 * \mathrm{x}+23.7, \mathrm{r}=0.88, \mathrm{SEE}=25.3, \mathrm{P}<$ 0.0001 and $\mathrm{y}=0.977^{*} \mathrm{x}+13.4, \mathrm{r}=0.88, \quad \mathrm{SEE}=28.3, \mathrm{P}<$ $0.0001)$.

As the Ao dp/dtmax is an ejection-phase parameter, so the LV ejection fraction (EF) is a widely used index of LV contractility during the ejection phase. Thus, it is possible that LVEF may also provide a way of accurately estimating LV $\mathrm{dp} / \mathrm{dtmax}$. To test this, we examined the relationship between LVEF and LV dp/dtmax in 26 patients in the disease group in whom LVEF could be calculated by ventriculography. The results, however, showed a poor correlation between $\mathrm{LVEF}$ and LV dp/dtmax ( $\mathrm{r}=0.04, \mathrm{P}=\mathrm{NS})$ in contrast to the highly significant correlation between Ao dp/dtmax and LV $\mathrm{dp} / \mathrm{dtmax}(\mathrm{r}=0.59, \mathrm{P}<0.005)$. Thus, although both Ao dp/dtmax and LVEF are ejection-phase parameters, Ao dp/dtmax has a significant superiority in estimating $\mathrm{LV} \mathrm{dp} / \mathrm{dtmax}$.

\section{Discussion}

The present study has demonstrated for the first time a close correlation between Ao dp/dtmax and LV dp/dtmax, with both $\mathrm{Zc}$ and MAP acting as interactive factors, and that such interactions are consistent among patients despite marked differences in underlying cardiac disease, contractile state, and HR. Using such a relationship, LV dp/dtmax was accurately predicted by Ao dp/dtmax in pediatric patients with various cardiovascular diseases and conditions. These results suggest the possibility that $\mathrm{LV}$ contractility can be noninvasively assessed by Ao dp/dtmax-derived LV dp/dtmax, helping broaden the bedside clinical applicability of LV dp/dtmax.

\section{Determinants of the Ao dp/dtmax $-\mathrm{LV} \mathrm{dp/dtmax}$ Relationship}

For a given blood flow ejected into the arterial system, the ascending rim of the aortic pressure trajectory is determined by the mechanical properties of the arterial bed, including Zc, Rt, and C. ${ }^{13}$ Figures $\mathbf{5 A}-\mathbf{C}$ illustrates the effects of changes in each property on the aortic pressure waveform. An increase in $\mathrm{Zc}$ causes a rise in peak aortic pressure without a change in minimum (diastolic) pressure, whereas an increase in Rt results in increases in both peak and minimum pressure. On the other hand, an increase in $\mathrm{C}$ increases the maximum pressure, but decreases the minimum pressure, without a change in mean pressure. Therefore, each of these vascular properties should theoretically affect the Ao dp/dtmax $-\mathrm{LV}$ dp/dtmax relationship by changing Ao dp/dtmax even when LV contractility ( $\mathrm{LV}$ dp/dtmax) is unchanged. In addition, changes in HR alone without changes in cardiac output or vascular properties also change the pulse pressure (Figure 5D), and thereby potentially affect the Ao dp/dtmax - LV dp/dtmax relationship. However, in the in-vivo human heart, changes in each property in turn affect ventricular ejection such that increases in $\mathrm{Zc}$ and $\mathrm{Rt}$ and a decrease in $\mathrm{C}$ significantly reduce ventricular ejection. Using an isolated canine heart that enables evaluation of the independent and quantitative effects of changes in vascular properties on ventricular ejection, Sunagawa et al demonstrated that $\mathrm{Rt}$ is a major determinant of stroke volume, whereas $\mathrm{Zc}$ and $\mathrm{C}$ had a significant but only minimal effect on ejection. ${ }^{14}$ Therefore, increases in Rt should reduce stroke volume and thereby counteract the effect of Rt change on the rise in Ao dp/dtmax. MAP represents the result of the interaction between $\mathrm{Rt}$ and stroke volume, with HR effects also incorporated; which may explain the reason for MAP, but not Rt or HR per se, being an important determinant of the Ao-LV dp/dtmax relationship. The reason why $\mathrm{Zc}$, but not $\mathrm{C}$, significantly affects the 
Ao-LV dp/dtmax relationship may also be related to the balance between their effects on aortic pressure trajectory and those on ventricular ejection (changes in Ao dp/dtmax outweigh the changes in ventricular ejection when $\mathrm{Zc}$ changes, whereas changes in Ao dp/dtmax are counterbalanced by changes in ejection when $\mathrm{C}$ changes).

Importantly, the relationship between the Ao-LV dp/dtmax ratio and vascular loading properties (Zc and MAP) was maintained irrespective of the underlying cardiac disease and conditions. The ventricular-vascular interaction is operational in the in-vivo working heart to determine the net hemodynamic performance under both physiologic and pathologic conditions. The Ao dp/dtmax $-\mathrm{LV}$ dp/dtmax relationship represented by equation 1 can be viewed as an expression of such an interaction, because the equation indicates that the ventricular property ( $\mathrm{LV} \mathrm{dp/dtmax)} \mathrm{and} \mathrm{the}$ vascular loading properties (right side of equation 1) determine the net hemodynamic consequence (Ao dp/dtmax). Thus, it appears to be a valid conclusion that the present results indicate a consistent relationship between Ao-LV $\mathrm{dp} / \mathrm{dtmax}$ ratio and vascular loading/mechanical properties among patients with different pathophysiologies. The applicability of equations 1 and 2 to the present VSD patients who had a shunt flow to the pulmonary artery from the LV would directly support this. In VSD patients with a large shunt, one may speculate that for a given LV contractility (LV dp/dtmax), Ao dp/dtmax may be lower, because of shunt flow through the VSD, than would be expected without a shunt. However, in such conditions, vascular stiffness to maintain blood pressure would also keep Ao dp/dtmax relatively unchanged for a given LV dp/dtmax, explaining the equality of equations 1 and 2 in VSD patients.

\section{Clinical Implications}

The central aortic pressure can be reasonably estimated by noninvasive tonometric measurement of the carotid artery pressure waveform combined with cuff-measured brachial arterial blood pressure. ${ }^{15,16}$ The central aortic pressure can also be reconstructed from peripheral arterial pressure measurements by utilizing the transfer function between each pressure waveform. ${ }^{17,18}$ In addition, umbilical artery catheterization often performed in critically ill neonates provides continuous monitoring of central aortic pressure (there is a close correlation between descending Ao dp/dtmax and ascending Ao dp/dtmax; descending Ao dp/dtmax =0.91 $\times$ Ao $\mathrm{dp} / \mathrm{dtmax}+79, \mathrm{R}=0.93, \mathrm{SEE}=61, \mathrm{n}=34$, unpublished data from our laboratory). Therefore, incorporating the present result of a consistent relationship between the Ao dp/dtmax/ $\mathrm{LV} \mathrm{dp/dtmax} \mathrm{ratio} \mathrm{and} \mathrm{vascular} \mathrm{loading} \mathrm{properties} \mathrm{into} \mathrm{the}$ noninvasively estimated central aortic pressure, together with simultaneously measurement of ascending aortic flow by echocardiography, is a novel method of noninvasive calculation of $\mathrm{LV}$ dp/dtmax.

Various methods for noninvasive estimation of $\mathrm{LV}$ $\mathrm{dp} / \mathrm{dtmax}$ have been proposed. ${ }^{19-21}$ They largely depend on Doppler signals of mitral insufficiency, and thus limiting their applicability to patients with mitral regurgitation. Our method is clearly advantageous in this regard because of its wide applicability. The method proposed by Rhodes et al only used diastolic blood pressure and isovolumic contraction time and thus did not require the presence of mitral insufficiency. ${ }^{3}$ Compared with their method, our approach requires somewhat complex calculations of impedance data and the derivative of aortic pressure, but such complexity could easily be circumvented by developing a computerized automated calculation algorithm. In addition, our method offers useful information about the ventricular-vascular interaction, by providing not only LV dp/dtmax but also vascular loading properties, and thus helps broaden the pathophysiological and therapeutic understanding in the clinical setting. Alternatively, as shown in Figures 3B and 4B, LV dp/dtmax can also be reasonably estimated from Ao $\mathrm{dp} / \mathrm{dtmax}$ even without the calculation of Zc (equation 2). Although this alternative produced a slightly higher SEE compared with using both MAP and Zc, the slope and intercept of regression were closer to unity and zero by this method than by the method using both MAP and Zc. Therefore, this method could be a useful surrogate and improve the clinical feasibility of estimating LV dp/dtmax when $\mathrm{Zc}$ is not obtainable. On the other hand, it should be noted that several factors are involved in the estimation of LV $\mathrm{dp} / \mathrm{dtmax}$ from Ao dp/dtmax and therefore the present method is not a direct alternative for the measurement of $\mathrm{LV}$ dp/dtmax.

\section{Study Limitations}

There are several limitations in the present study that deserve further discussion. Although we used Kawasaki disease patients as a control group, they may not be truly normal. However, these patients did not have heart failure or shunting disorders, and had normal cardiac function and structure on routine echocardiographic check-up. Furthermore, the vascular properties of the Kawasaki disease patients considerably differed from those of the disease group. Therefore, we believe that the Kawasaki disease patients can be regarded as a control group for the purpose of the present study.

Although the present results indicate that LV contractility can be noninvasively assessed by the Ao dp/dtmax-derived $\mathrm{LV} \mathrm{dp/dtmax,} \mathrm{this} \mathrm{remains} \mathrm{to} \mathrm{be} \mathrm{validated} \mathrm{in} \mathrm{a} \mathrm{future} \mathrm{study,}$ including the accuracy of Ao dp/dtmax derived by tonometric measurement.

The number of patients studied was limited and the diagnoses were biased. Therefore, it remains to be elucidated whether the relationship obtained in the present study is applicable to other, more seriously unwell pediatric cardiac patients (eg, those with dilated cardiomyopathy or complex congenital heart disease patients, including those with a single ventricle), in whom a sophisticated assessment of LV contractility would be more necessary than in the present study population.

In the present study, we could not test the effect of acute change in afterload on the Ao- $\mathrm{LV}$ dp/dtmax ratio for ethical reasons, because an acute increase in afterload may jeopardize the patient's condition. Although our disease group patients had a relatively broad range of afterload conditions, it should be noted that large changes in afterload may potentially affect the Ao-LV dp/dtmax relationship, because the relationship between the vascular mechanical/ loading properties and the Ao-LV dp/dtmax ratio (equations 1 and 2) may not be linear, particularly where afterload changes acutely and extensively. Although afterload-independence is a major advantage of $\mathrm{LV} \mathrm{dp/dtmax}$, our methods estimate LV dp/dtmax by utilizing afterload data. Because several contractile indices are available using ventricularafterload relationships, $\mathrm{LV} \mathrm{dp} / \mathrm{dtmax}$ obtained from the present method should be regarded as 1 such index, but not as a direct alternative to afterload-independent $\mathrm{LV} \mathrm{dp/dtmax.}$

More than 3 decades ago, Taylor et al $^{22}$ hoped to use Ao

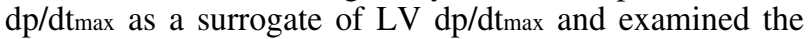


relationship between these 2 variables. They indeed found a close correlation, but also found considerable variation in the relationship under different test conditions, including varied afterload conditions, altered ventricular contractility, and during exercise. Unfortunately, they did not attempt to find the factors that induce such variance and consequently abandoned the use of Ao dp/dtmax as a potential indicator of LV contractility. The present study has provided a clear answer to their question raised decades ago but left unanswered.

\section{Conclusion}

The Ao dp/dtmax-LV dp/dtmax relationship correlated consistently through the interaction of arterial mechanical and loading properties. Using such a relationship, LV dp/dtmax can be predicted accurately by Ao dp/dtmax regardless of the background cardiovascular disease, potentially providing a method for noninvasive determination of LV contractility.

\section{Acknowledgment}

Grant support: a national grant (no. 8025127) from the Japan Society for the Promotion of Science and Medical Research, Grants from Nipro Corporation, Kawano Memorial Foundation, and Tenshindo Medical Institution.

\section{Disclosure}

None.

\section{References}

1. Quinones MA, Gaasch WH, Alexander JK. Influence of acute changes in preload, afterload, contractile state and heart rate on ejection and isovolumic indices of myocardial contractility in man. Circulation 1976; 53: 293-302.

2. Little WC. The left ventricular $\mathrm{dP} / \mathrm{dtmax}$-end-diastolic volume relation in closed-chest dogs. Circ Res 1985; 56: 808-815.

3. Rhodes J, Udelson JE, Marx GR, Schmid CH, Konstam MA, Hijazi $\mathrm{ZM}$, et al. A new noninvasive method for the estimation of peak dP/dt. Circulation 1993; 88: 2693-2699.

4. Senzaki H, Isoda T, Paolocci N, Ekelund U, Hare JM, Kass DA. Improved mechanoenergetics and cardiac rest and reserve function of in vivo failing heart by calcium sensitizer EMD-57033. Circulation 2000; 101: 1040-1048.

5. Senzaki H, Paolocci N, Gluzband YA, Lindsey ML, Janicki JS, Crow MT, et al. beta-blockade prevents sustained metalloproteinase activation and diastolic stiffening induced by angiotensin II combined with evolving cardiac dysfunction. Circ Res 2000; 86: 807-815.

6. Hagiwara S, Iwasaka H, Uchino T, Noguchi T. High mobility group box 1 induces a negative inotropic effect on the left ventricle in an isolated rat heart model of septic shock: A pilot study. Circ J 2008; 72: $1012-1017$.

7. Mercanoglu G, Safran N, Gungor M, Pamukcu B, Uzun H, Sezgin C, et al. The effects of nebivolol on apoptosis in a rat infarct model. Circ J 2008; 72: 660-670.

8. Kiuchi K, Yoshida A, Fukuzawa K, Takano T, Kanda G, Takami K, et al. Identification of the right ventricular pacing site for cardiac resynchronization therapy (CRT) guided by electroanatomical mapping (CARTO). Circ J 2007; 71: 1599-1605.

9. Senzaki H, Chen $\mathrm{CH}$, Masutani S, Taketazu M, Kobayashi J, Kobayashi T, et al. Assessment of cardiovascular dynamics by pressure-area relations in pediatric patients with congenital heart disease. J Thorac Cardiovasc Surg 2001; 122: 535-547.

10. Senzaki H, Chen CH, Ishido H, Masutani S, Matsunaga T, Taketazu M, et al. Arterial hemodynamics in patients after Kawasaki disease. Circulation 2005; 111: 2119-2125.

11. Senzaki H, Isoda T, Ishizawa A, Hishi T. Reconsideration of criteria for the Fontan operation: Influence of pulmonary artery size on postoperative hemodynamics of the Fontan operation. Circulation 1994; 89: $266-271$.

12. Liu Z, Brin KP, Yin FC. Estimation of total arterial compliance: An improved method and evaluation of current methods. Am J Physiol 1986; 251: H588-H600.

13. Nichols W, O'Rourke M. Vascular impedance. In: McDonald, editor. Blood flow in arteries. London: Edward Arnold; 1990; 283-329.

14. Sunagawa K, Maughan WL, Burkhoff D, Sagawa K. Left ventricular interaction with arterial load studied in isolated canine ventricle. Am J Physiol 1983; 245: H773-H780.

15. Kelly R, Fitchett D. Noninvasive determination of aortic input impedance and external left ventricular power output: A validation and repeatability study of a new technique. J Am Coll Cardiol 1992; 20: 952-963.

16. Marcus RH, Korcarz C, McCray G, Neumann A, Murphy M, Borow $\mathrm{K}$, et al. Noninvasive method for determination of arterial compliance using Doppler echocardiography and subclavian pulse tracings: Validation and clinical application of a physiological model of the circulation. Circulation 1994; 89: 2688-2699.

17. Chen CH, Nevo E, Fetics B, Pak PH, Yin FC, Maughan WL, et al. Estimation of central aortic pressure waveform by mathematical transformation of radial tonometry pressure: Validation of generalized transfer function. Circulation 1997; 95: 1827-1836.

18. Fetics B, Nevo E, Chen CH, Kass DA. Parametric model derivation of transfer function for noninvasive estimation of aortic pressure by radial tonometry. IEEE Trans Biomed Eng 1999; 46: 698-706.

19. Bargiggia GS, Bertucci C, Recusani F, Raisaro A, de Servi S, ValdesCruz LM, et al. A new method for estimating left ventricular $\mathrm{dP} / \mathrm{dt}$ by continuous wave Doppler-echocardiography: Validation studies at cardiac catheterization. Circulation 1989; 80: 1287-1292.

20. Chen C, Rodriguez L, Guerrero JL, Marshall S, Levine RA, Weyman $\mathrm{AE}$, et al. Noninvasive estimation of the instantaneous first derivative of left ventricular pressure using continuous-wave Doppler echocardiography. Circulation 1991; 83: 2101-2110.

21. Chung N, Nishimura RA, Holmes DR Jr, Tajik AJ. Measurement of left ventricular dp/dt by simultaneous Doppler echocardiography and cardiac catheterization. J Am Soc Echocardiogr 1992; 5: 147-152.

22. Taylor SH, Snow HM, Linden RJ. Relationship between left ventricular and aortic dP-dt (max). Proc R Soc Med 1972; 65: 550-552. 